\title{
Acute erythroid leukemia with $<20 \%$ bone marrow blasts is clinically and biologically similar to myelodysplastic syndrome with excess blasts
}

\author{
Sa A Wang ${ }^{1}$, Keyur P Patel ${ }^{1}$, Olga Pozdnyakova ${ }^{2}$, Jie Peng ${ }^{1}$, Zhuang Zuo ${ }^{1}$, Paola Dal Cin ${ }^{2}$, \\ David P Steensma ${ }^{3}$ and Robert P Hasserjian ${ }^{4}$ \\ ${ }^{1}$ Department of Hematopathology, The University of Texas MD Anderson Cancer Center, Houston, TX, USA; \\ ${ }^{2}$ Department of Pathology, Brigham and Women's Hospital, Boston, MA, USA; ${ }^{3}$ Department of Medical \\ Oncology, Dana-Farber Cancer Institute, Boston, MA, USA and ${ }^{4}$ Department of Pathology, Massachusetts \\ General Hospital, Boston, MA, USA
}

\begin{abstract}
In acute erythroleukemia, erythroid/myeloid subtype, blasts usually comprise $5-19 \%$ of total bone marrow cells, similar to the myelodysplastic syndrome subtype refractory anemia with excess blasts; recent studies have raised the question if acute erythroleukemia should be considered as a myelodysplastic syndrome subtype. We reviewed 77 de novo acute erythroleukemia and 279 de novo refractory anemia with excess blasts from three large medical centers. Compared to refractory anemia with excess blasts, acute erythroleukemia patients had higher total bone marrow blasts, lower platelets, hemoglobin, and absolute neutrophil counts, with more patients being assigned a very-poor-karyotype risk and very-high Revised International Prognostic Scoring System score. Induction chemotherapy was administered to $55 \%$ of acute erythroleukemia patients, but was not associated with longer overall survival compared to acute erythroleukemia patients treated with lower-intensity therapies or supportive care $(P=0.44)$. In multivariable analysis of all patients, Revised International Prognostic Scoring System very high $(P<0.0001)$ or high $(P=0.005)$ risk, but not a diagnosis of acute erythroleukemia $(P=0.30)$, were independent risk factors for shorter overall survival. Our data show that acute erythroleukemia patients have similar risk-adjusted outcome to refractory anemia with excess blasts patients and do not appear to gain survival advantage with acute myeloid leukemia-type induction chemotherapy. These data suggest that acute erythroleukemia, erythroid/myeloid subtype with $<20 \%$ blasts may be more appropriately classified as refractory anemia with excess blasts rather than as an acute myeloid leukemia subtype.
\end{abstract}

Modern Pathology (2016) 29, 1221-1231; doi:10.1038/modpathol.2016.118; published online 22 July 2016

Acute erythroleukemia, erythroid/myeloid subtype is a rare acute myeloid leukemia subtype in the 2008 World Health Organization Classification of myeloid neoplasms. ${ }^{1}$ The criteria for acute erythroleukemia require the presence of $\geq 50 \%$ erythroid cells and $\geq 20 \%$ blasts of non-erythroid cells in bone marrow. In cases that meet this definition, bone marrow blasts typically range from 5 to $19 \%$ of total marrow cells, similar to the myelodysplastic syndrome subtype refractory anemia with excess blasts. Acute

Correspondence: Dr RP Hasserjian, MD, Department of Pathology, WRN244, Massachusetts General Hospital, 55 Fruit Street, Boston 02114, MA, USA.

E-mail: rhasserjian@partners.org

Received 10 March 2016; revised 11 May 2016; accepted 29 May 2016; published online 22 July 2016 erythroleukemia was first named as a variant of acute myeloid leukemia by the French-American-British (FAB) classification in 1985, requiring myeloblasts $\geq 30 \%$ of the non-erythroids in cases with $\geq 50 \%$ bone marrow erythroid cells. ${ }^{2}$ In the 2001 World Health Organization Classification of Myeloid Neoplasms, the minimum blast count for all types of acute myeloid leukemia was lowered from 30 to $20 \%$. This change accordingly lowered the blast count defining acute erythroleukemia to $20 \%$ of the non-erythroid cells ${ }^{1}$ and thus reclassified many myelodysplastic syndrome cases with a major erythroid component to the acute erythroleukemia category. The historical reason behind placing a myeloid neoplasm with $<20 \%$ blasts under acute myeloid leukemia was the perception that the adverse features of acute erythroleukemia could not 
be properly captured by the absolute myeloblast percentage in the setting of the dominant erythroid component of this disease. ${ }^{2}$

In enumerating the blast proportion of bone marrow cells (even when counting 500 cells), ${ }^{3}$ minor differences in blast percentages may reclassify cases from myelodysplastic syndrome to acute myeloid leukemia; this uncertainty is magnified in cases with $\geq 50 \%$ erythroid cells, since the exclusion of erythroids produces a much smaller denominator for blast enumeration. While erythroid predominance ( $\geq 50 \%$ of bone marrow erythroids) is seen in about $15 \%$ of myelodysplastic syndrome cases, ${ }^{4}$ in such cases the blast count is enumerated as a proportion of all nucleated bone marrow cells rather than just the non-erythroid component. About one-third of acute erythroleukemia cases evolve from an antecedent myelodysplastic syndrome, and a slight increase in blast count in erythroid-predominant myelodysplastic syndrome cases can lead to re-classification as acute erythroleukemia, which would be considered as disease progression even though this small difference may not be clinically meaningful ${ }^{5,6}$. The diagnostic challenges with respect to acute erythroleukemia and refractory anemia with excess blasts were highlighted in a 2013 workshop hosted by the Society of Hematopathology: in many of the cases submitted to workshop, a definitive separation between acute erythroleukemia and refractory anemia with excess blasts was felt to be difficult by the submitters and the expert panel. ${ }^{7}$

The diagnostic challenges in distinguishing acute erythroleukemia from refractory anemia with excess blasts and the therapeutic implications of a diagnosis of acute leukemia demand a better definition of acute erythroleukemia. Recent studies have suggested that acute erythroleukemia may be closer to myelodysplastic syndrome than to other subtypes of acute myeloid leukemia in terms of its genetic characteristics and clinical behavior. ${ }^{8-10}$ However, many prior acute erythroleukemia series have included therapy-related myeloid neoplasms or cases known to have progressed from pre-existing myelodysplastic syndromes, which often show different molecular genetic characteristics and prognosis from their de novo counterparts. Until now, the features of de novo acute erythroleukemia have not been directly compared with de novo myelodysplastic syndrome with a similar absolute bone marrow blast percentage. While a diagnosis of acute myeloid leukemia is not a mandate to treat with induction chemotherapy, a pathologic diagnosis of an acute leukemia often prompts clinicians to act differently from a myelodysplastic syndrome diagnosis, which is usually not treated with up-front induction chemotherapy. The rarity of acute erythroleukemia precludes prospective randomized trials to evaluate response to acute myeloid leukemia vs myelodysplastic syndrometype therapies such as DNA hypomethylating agents, thus the optimal therapeutic approach to acute erythroleukemia is uncertain.
In this retrospective study, we collected the largest series of de novo acute erythroleukemia compiled to date and compared these cases to a large series of primary refractory anemia with excess blasts diagnosed and treated during the same time period at three large medical centers in the United States. We examined the clinical and genetic features of acute erythroleukemia in comparison to refractory anemia with excess blasts and tested if acute erythroleukemia patient risk could be accurately stratified by the Revised International Prognostic Scoring System scheme for myelodysplastic syndrome. We also compared treatment approaches of acute erythroleukemia to refractory anemia with excess blasts and assessed outcome of patients treated with different modalities. Our aim was to identify similarities and differences between the presenting features, treatment approach, and outcome of acute erythroleukemia in comparison to high-risk myelodysplastic syndrome.

\section{Materials and methods}

\section{Patients}

We reviewed pathology archives of three institutions (Massachusetts General Hospital, Brigham and Women's Hospital, and MD Anderson Cancer Center) for newly diagnosed cases of refractory anemia with excess blasts (including both refractory anemia with excess blasts- 1 and -2 by the 2008 World Health Organization Classification criteria) or acute erythroleukemia (erythroid/myeloid subtype) in adults (age $\geq 18$ years) between 1 January 2003 and 7 January 2014. Patients with a history of any antecedent myeloid neoplasm and known or suspected therapyrelated myeloid neoplasms were excluded. Cases were classified according to the 2008 World Health Organization Classification criteria, based on counts of aspirate smears or touch preparations of bone marrow, peripheral blood, and presence or absence of Auer rods, and were assigned to three groups: refractory anemia with excess blasts without erythroid predominance (MDS-typical group), fulfilling criteria for refractory anemia with excess blasts-1 or -2 and with $<50 \%$ bone marrow erythroid precursors; erythroid-predominant refractory anemia with excess blasts (MDS-erythroid group), fulfilling criteria for refractory anemia with excess blasts- 1 or -2 and with $\geq 50 \%$ bone marrow erythroid precursors; and acute erythroleukemia (AEL group), fulfilling criteria for a diagnosis of acute erythroleukemia, erythroid-myeloid subtype $(\geq 50 \%$ bone marrow erythroid precursors and myeloblasts comprising $\geq 20 \%$ of the non-erythroid bone marrow cells cells), but with blasts comprising $<20 \%$ of all bone marrow cells and peripheral blood leukocytes.

Clinical information at presentation and follow-up information, including treatment modalities and overall survival, were retrieved from the electronic 
medical records at each institution. As this study was retrospective, treatment decisions were based on the practice at each respective institution where the cases were diagnosed. Treatments were categorized as supportive care only (including growth factors, steroids, or hydroxyurea ${ }^{11}$ ), low-intensity therapies other than hypomethylating agents (lenalidomide, low-dose cytarabine, and other cytotoxic or noncytotoxic investigational agents), hypomethylating agents (azacitidine and decitabine), induction therapy (standard induction therapy employing an anthracycline and cytarabine), and allogeneic stem cell transplant. This study was approved by the Institutional Review Boards of all participating institutions.

\section{Pathology}

Pathologic features recorded for each case included bone marrow cellularity, erythroid precursor count, and blast count. Blast count was based on counting 200 (peripheral blood) and 500 (bone marrow) cells, and expressed as a percentage of all cells and of nonerythroid cells in the bone marrow. Ring sideroblasts were counted on Perls-stained bone marrow aspirate smears and were expressed as the percentage of nucleated erythroid cells.

\section{Cytogenetics and Molecular Genetic Studies}

All cases with available karyotype were stratified according to the Comprehensive Cytogenetic Scoring System of the Revised International Prognostic Scoring System. ${ }^{12}$ Genomic DNA was extracted from bone marrow unfractionated cells or from fresh frozen tissue/bone marrow aspirate. Testing for mutations including NPM1, JAK2, CEBPA, KIT, and FLT3 was performed in a subset of cases as part of the routine clinical workup in the laboratory. Additional studies to evaluate the mutation status of TP53, IDH1, IDH2, JAK2, NRAS, KRAS, and KIT were performed in cases with available freshly archived material by next generation sequencing methods using a customized TruSeq Amplicon Cancer Panel and a MiSeq sequencer (Illumina Inc, San Diego, CA) ${ }^{13,14}$ at MD Anderson Cancer Center and using the clinically validated Snapshot-NGS-V1 assay ${ }^{15}$ and a MiSeq sequencer at Massachusetts General Hospital.

\section{Statistical Analysis}

Data in tables are expressed as medians (ranges) unless otherwise specified. Fisher's exact test and Mann-Whitney test were used to compare categorical and continuous variables between groups, respectively. Overall survival from diagnosis was estimated using the Kaplan and Meier method and the log-rank test was used to compare overall survival between groups. Multivariable subsequent to univariate Cox proportional hazards regression models were used to assess the impact of the patient groups, treatment modalities, and other risk factors on overall survival. A two-sided $P$-value of less than 0.05 was considered statistically significant.

\section{Results}

\section{Patient Characteristics}

A total of 77 cases of de novo acute erythroleukemia (AEL group) and 279 cases of de novo refractory anemia with excess blasts were identified. The latter group included 238 in the MDS-typical group (85\%) and 41 in the MDS-erythroid group (15\%). In all, 3/41 (7\%) of the MDS-erythroid and 138/238 (58\%) of the MDS-typical group were classified as refractory anemia with excess blasts-2, while the remainder were refractory anemia with excess blasts-1. The AEL group included one patient with $4 \%$ bone marrow blasts (and with blasts comprising $21 \%$ of the non-erythroids), the MDSerythroid group included four patients with 3-4\% bone marrow blasts and $\geq 2 \%$ peripheral blood blasts, and the MDS-typical group included five patients with $0-4 \%$ bone marrow blasts and $\geq 2 \%$ peripheral blood blasts and one patient with $4 \%$ bone marrow blasts and Auer rods; in all other patients, blasts were 5-19\% of the total bone marrow nucleated cells.

Patient characteristics, including age, sex, and peripheral blood and bone marrow features of all three groups, are shown in Table 1. Compared to MDStypical patients, AEL patients had significantly lower peripheral blood counts and higher marrow cellularity and bone marrow and blood blast percentages (Table 1): $67 / 77$ (87\%) of AEL patients compared to 124/238 (53\%) MDS-typical patients had $\geq 10 \%$ BM blasts $(P<0.0001)$. The Comprehensive Cytogenetic Scoring System karyotype risk and overall Revised International Prognostic Scoring System risk group distribution of the patient groups are shown in Figure 1. A higher proportion of both AEL patients $(P=0.04)$ and MDS-erythroid $(P=0.02)$ patients had Comprehensive Cytogenetic Scoring System verypoor-risk karyotype compared to MDS-typical patients. AEL patients also had a significantly higher overall Revised International Prognostic Scoring System score $(P=0.003)$ and a significantly larger proportion of Revised International Prognostic Scoring System veryhigh-risk patients $(P=0.003)$ compared to MDS-typical patients. Although TP53 mutations were more frequently seen in AEL (33\%) compared to MDS-typical $(21 \%)$ or MDS-erythroid (22\%) groups, these differences were not statistically significant. AEL had low incidences of FLT3 ITD and NPM1 mutations, which were not statistically significantly different from the similarly low incidences of these mutations seen in MDS-typical and MDS-erythroid groups (Table 1).

\section{Treatments}

Treatments given to the different patient groups are shown in Table 2. Among patients who did not 
Table 1 Comparison of clinicopathologic and genetic features between acute erythroid leukemia (AEL) with $<20 \%$ blasts and refractory anemia with excess blasts with (MDS-erythroid) and without (MDS-typical) erythroid predominance

\begin{tabular}{|c|c|c|c|c|c|c|}
\hline & \multirow[b]{2}{*}{$\begin{array}{c}A E L \\
(\mathrm{n}=77)\end{array}$} & \multirow{2}{*}{$\begin{array}{c}\text { MDS-erythroid } \\
(\mathrm{n}=41)\end{array}$} & \multirow[b]{2}{*}{$\begin{array}{c}\text { MDS-typical } \\
(\mathrm{n}=238)\end{array}$} & \multicolumn{3}{|c|}{$\mathrm{P}$ values } \\
\hline & & & & $\begin{array}{c}\text { AEL vs } \\
\text { MDS-typical }\end{array}$ & $\begin{array}{c}\text { AEL vS } \\
\text { MDS-erythroid }\end{array}$ & $\begin{array}{l}\text { MDS-erythroid } \\
\text { vs MDS-typical }\end{array}$ \\
\hline Age, years & $66(22-93)$ & $63(30-91)$ & $68.5(18-92)$ & 0.34 & 0.09 & 0.009 \\
\hline Males & $60 / 77(78 \%)$ & $30 / 41(73 \%)$ & $164 / 238(69 \%)$ & 0.15 & 0.65 & 0.71 \\
\hline White blood cell count, $\times 10^{9} / \mathrm{L}$ & $2.0(0.6-16.4)$ & $2.6(0.9-8.6)$ & $2.9(0.1-21.3)$ & 0.006 & 0.25 & 0.08 \\
\hline Absolute neutrophil count, $\times 10^{9} / \mathrm{L}$ & $0.8(0.1-7.1)$ & $0.9(0.1-5.8)$ & $1.2(0-10.3)$ & 0.002 & 0.18 & 0.27 \\
\hline Peripheral blood blast \%, mean & $2.8(0-18)$ & $1.0(0-7)$ & $1.6(0-18)$ & 0.015 & 0.10 & 0.96 \\
\hline Hemoglobin, g/dL & $9.1(4.9-17.5)$ & $9.7(7.7-15.8)$ & $9.4(5.0-13.6)$ & 0.04 & 0.02 & 0.24 \\
\hline Platelets, $\times 10^{9} / \mathrm{L}$ & $56(10-426)$ & $68(15-269)$ & $73(3-614)$ & 0.035 & 0.14 & 0.96 \\
\hline Bone marrow cellularity \% & $80(20-100)$ & $80(10-100)$ & $70(10-100)$ & 0.01 & 0.35 & 0.39 \\
\hline Bone marrow erythroid \% & $61(50-89)$ & $55(50-72)$ & $26.5(0-49)$ & $<0.0001$ & 0.002 & $<0.0001$ \\
\hline Bone marrow blast $\%$ of total cells, mean & $12.2(4-19)$ & $6.4(3-9)$ & $10.2(0-19)$ & $<0.0001$ & $<0.0001$ & $<0.0001$ \\
\hline Bone marrow blast \% of non-erythroids & $33(20-59)$ & $15(9-19.5)$ & $13(0-30.5)$ & $<0.0001$ & $<0.0001$ & 0.25 \\
\hline Ring sideroblasts $\geq 15 \%$ & $22 / 56(39 \%)$ & $9 / 29(31 \%)$ & $14 / 133(10 \%)$ & 0.0001 & 0.49 & 0.008 \\
\hline IPSS-R very poor karyotype & $28 / 76(37 \%)$ & $17 / 40(43 \%)$ & $57 / 236(24 \%)$ & 0.04 & 0.56 & 0.02 \\
\hline$-5 / \operatorname{del}(5 q)$ & $27 / 76(36 \%)$ & $17 / 40(43 \%)$ & $67 / 236(28 \%)$ & 0.25 & 0.54 & 0.09 \\
\hline$-7 / \operatorname{del}(7 q)$ & $20 / 76(26 \%)$ & $21 / 40(53 \%)$ & $55 / 236(23 \%)$ & 0.64 & 0.007 & 0.0004 \\
\hline IPSS-R numerical score & $6.5(3-10)$ & $6(3-8.5)$ & $5.5(2-10)$ & 0.003 & 0.09 & 0.91 \\
\hline IPSS-R very high risk group & $42 / 76(55 \%)$ & $18 / 40(45 \%)$ & $83 / 233(36 \%)$ & 0.003 & 0.33 & 0.29 \\
\hline \multicolumn{7}{|l|}{ Mutation status } \\
\hline FLT3 ITD mutation & $2 / 73(3 \%)$ & $0 / 20(0 \%)$ & $3 / 105(3 \%)$ & 1 & 1 & 1 \\
\hline NPM1 mutation & $3 / 65(5 \%)$ & $0 / 16(0 \%)$ & $1 / 95(1 \%)$ & 0.30 & 1 & 1 \\
\hline$C E B P A$ mutation $^{\mathrm{a}}$ & $3 / 34(9 \%)$ & $0 / 9(0 \%)$ & $6 / 119(9 \%)$ & n.d. & n.d. & n.d. \\
\hline TP53 mutation & 15/45 (33\%) & $2 / 9(22 \%)$ & 18/85 (21\%) & 0.14 & 0.7 & 1 \\
\hline IDH1/IDH2 mutation & $3 / 48(6 \%)$ & $0 / 12(0 \%)$ & $10 / 86(12 \%)$ & n.d. & n.d. & n.d. \\
\hline DNMT3A mutation & $3 / 37(8 \%)$ & $0 / 8(0 \%)$ & $2 / 68(3 \%)$ & n.d. & n.d. & n.d. \\
\hline NRAS/KRAS mutation & $5 / 59(8 \%)$ & $1 / 18(6 \%)$ & $9 / 99(9 \%)$ & n.d. & n.d. & n.d. \\
\hline KIT mutation & $2 / 55(4 \%)$ & $0 / 16(0 \%)$ & 0/94 (0\%) & n.d. & n.d. & n.d. \\
\hline JAK2 mutation & $2 / 35(6 \%)$ & $0 / 12(0 \%)$ & $0 / 66(0 \%)$ & n.d. & n.d. & n.d. \\
\hline
\end{tabular}

Abbreviation: IPSS-R, Revised International Prognostic Scoring System.

a All were single CEBPA mutations.

receive stem cell transplant, AEL patients were significantly more likely to be treated with induction chemotherapy compared to MDS-typical patients despite their similar age $(P=0.01)$, and showed a trend toward lower likelihood of treatment with hypomethylating agents than MDS-typical patients $(P=0.07)$. As this was a retrospective study, we sought to analyze differences in the presenting features of AEL patients who received induction chemotherapy (with or without subsequent stem cell transplant) that might have influenced treatment choice. AEL patients treated with induction chemotherapy were significantly younger than those treated with lower-intensity therapies or supportive care (median age 62 vs 73 years, $P<0.0001$ ) and had a trend toward higher peripheral blood blast count $(P=0.08)$ and higher Revised International Prognostic Scoring System score $(P=0.09)$; there was no significant association between bone marrow blasts (either as a proportion of all cells or of non-erythroid cells), blood counts, sex, institution where the patient was treated, or very-high-risk karyotype and the use of induction chemotherapy in AEL patients.

There were no significant differences in the proportions of AEL, MDS-erythroid, and MDStypical patients treated with stem cell transplant.
However, 23/26 (88\%) AEL patients compared to 5/19 (26\%) MDS-erythroid $(P<0.0001)$ and $28 / 84$ (33\%) MDS-typical $(P<0.0001)$ patients received induction chemotherapy prior to stem cell transplant. The median times between diagnosis and stem cell transplant were 6.4 months (range 2.0-15.1 months) for AEL patients, 5.6 months (range 2.6-35.0 months) for MDS-erythroid patients, and 5.3 months (range 1.0-73.1 months) for MDS-typical patients, which were not significantly different $(P=0.75)$.

\section{Progression to Acute Myeloid Leukemia in MDS Patients}

At the time of latest followup, 18/41 (44\%) of MDSerythroid and 114/238 (48\%) of MDS-typical patients had transformed to acute myeloid leukemia (Table 2). Acute myeloid leukemia arising in MDS-erythroid patients was more likely to be of the acute erythroleukemia subtype compared to acute myeloid leukemia arising in MDS-typical patients $(P=0.02)$ (Table 2). There was no statistically significant difference in the proportion of acute myeloid leukemia post-MDS-erythroid vs acute myeloid leukemia post-MDS-typical patients treated with induction chemotherapy (data not shown). 
a

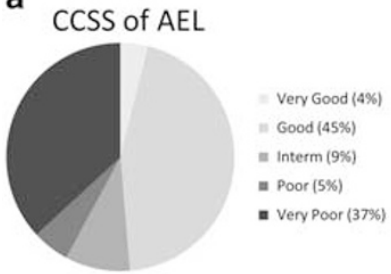

b

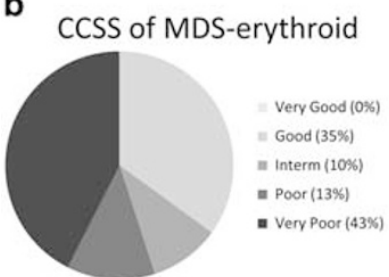

c CCSS of MDS-typical

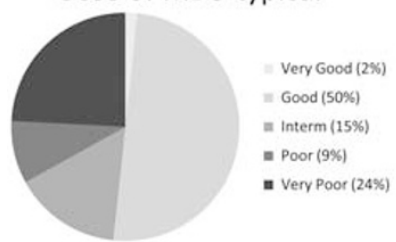

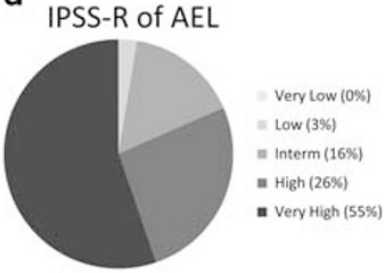

e

IPSS-R of MDS-erythroid

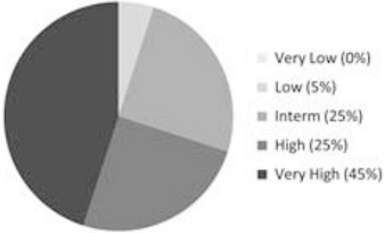

f

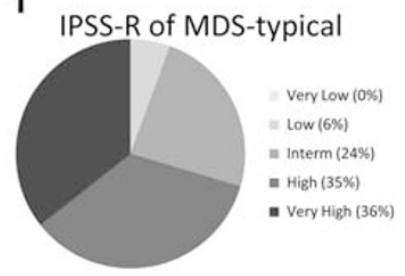

Figure 1 Distribution of Comprehensive Cytogenetic Scoring System (CCSS) karyotype risk groups among AEL (a), MDSerythroid (b), and MDS-typical (c) and distribution of overall Revised International Prognostic Scoring System risk grouping (IPSS-R) among AEL (d), MDS-erythroid (e), and MDS-typical (f) There is a significantly larger proportion of very-poor-risk karyotypes in AEL $(P=0.04)$ and MDS-erythroid $(P=0.02)$ compared to MDS-typical, and a larger proportion of very high Revised International Prognostic Scoring System risk group patients in AEL $(P=0.003)$ compared to MDS-typical.

\section{Outcome}

The median follow-up time of all patients was 15.8 months (range 0.3-135 months) and of living patients was 33.7 months. The median overall survivals of AEL, MDS-erythroid, and MDS-typical patients were 14.0, 16.4, and 18.5 months, respectively (Figure 2a). The median overall survival for AEL, MDS-erythroid, and MDS-typical patients $\leq 65$ years old were 14.5, 15.7, and 23.1 months, respectively. AEL patients showed a trend toward shorter overall survival compared to MDS-typical in all patients $(P=0.14)$ and in patients $\leq 65$ years $(P=0.15)$ that did not reach statistical significance. Since MDS-erythroid and MDS-typical patients showed similar patterns of treatment and outcome, these two groups were considered together (MDS) in the subsequent outcome analysis. Univariate analysis of all patients $(n=356)$ identified older age (as a continuous variable, $P<0.0001$ ), very poor Comprehensive Cytogenetic Scoring System karyotype risk $(P<0.0001)$, and Revised International Prognostic Scoring System very-high-risk categories $(P<0.0001$; compared to combined low/intermediate categories) as significant risk factors for shorter

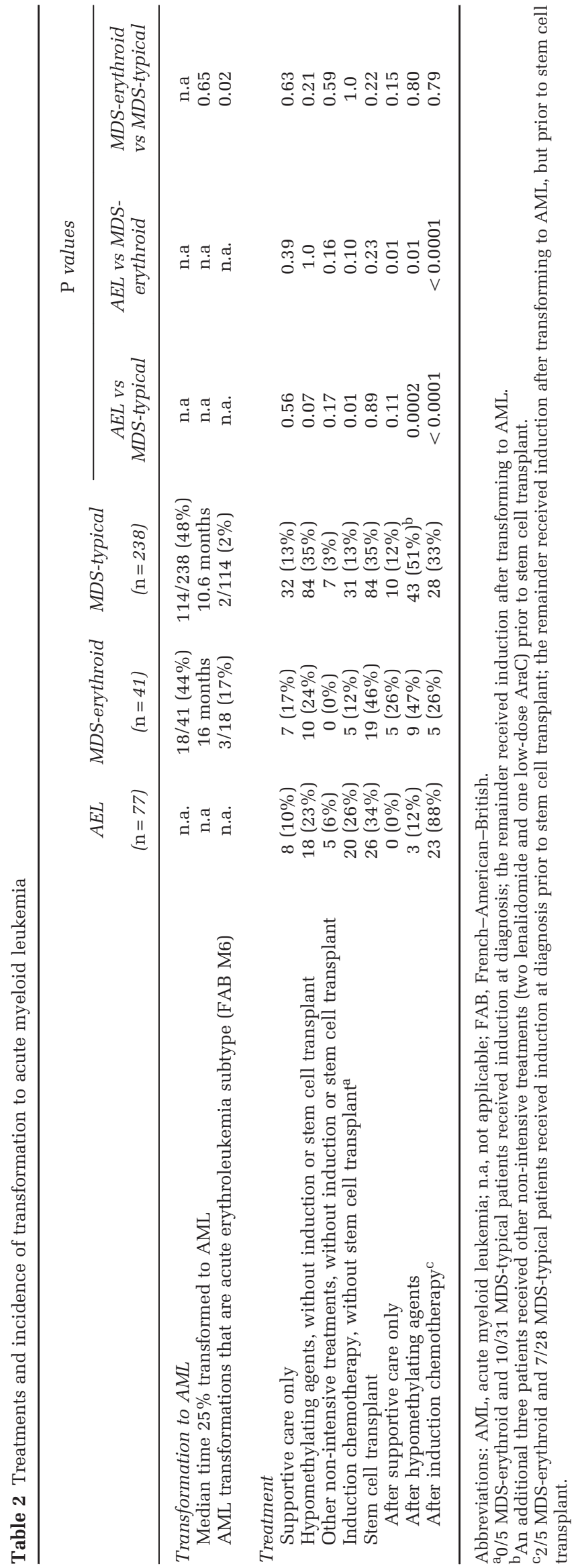




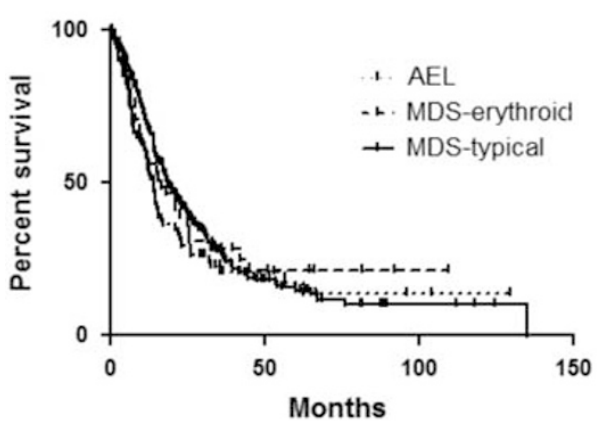

C
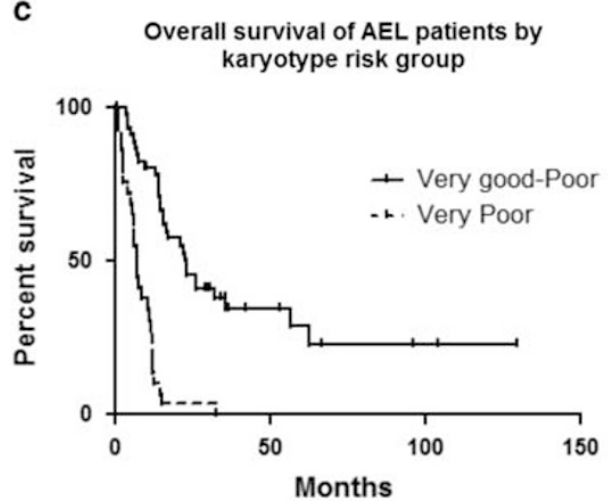
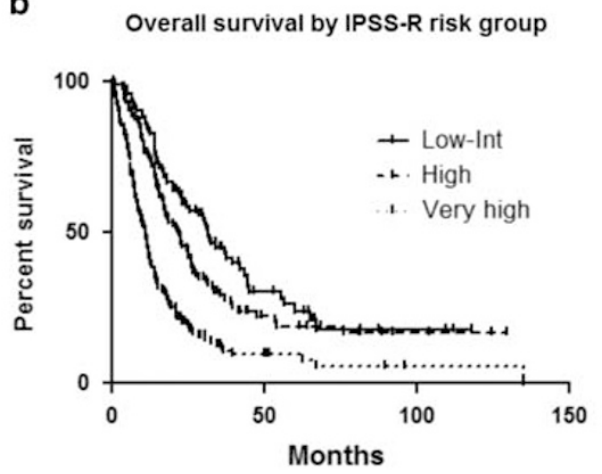

d

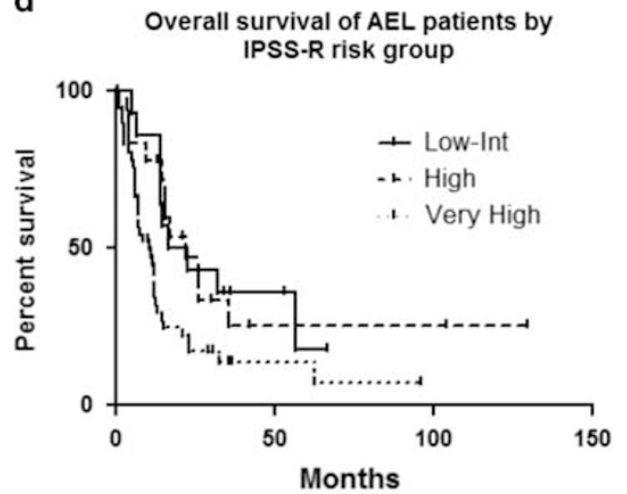

Figure 2 Overall survival of patients according to diagnosis group, karyotype risk, and Revised International Prognostic Scoring System risk group. (a) AEL patients (median overall survival 14 months) show a trend toward shorter overall survival compared to MDS-typical patients (median overall survival 18.5 months, $P=0.14$ ), and is similar to the overall survival of MDS-erythroid patients (median 16.4 months, $P=0.37$ ). (b) Considering all patients with available Revised International Prognostic Scoring System information ( $n=349$ ), overall survival of patients with low-intermediate risk (median 31.7 months) is borderline longer than that of patients with high risk (median 22.1 months, $P=0.06$ ), which is significantly longer than those with very high risk (median 11.0 months, $P<0.0001$ ). (c) Stratifying AEL patients by Comprehensive Cytogenetic Scoring System karyotype risk, patients with very poor risk (median overall survival 6.9 months) have significantly shorter overall survival compared to those with lower-risk karyotypes (median overall survival 22.8 months, $P<0.0001$ ). (d) AEL patients with very high Revised International Prognostic Scoring System risk have significantly shorter overall survival (median 10.5 months) compared to those with high risk (median overall survival 22.1 months, $P=0.02$ ), which is not significantly different from those with low-intermediate risk (median overall survival 19.6 months, $P=0.96$ ).

overall survival, with a trend of shorter survival for Revised International Prognostic Scoring System high risk $(P=0.06)$ (Figure 2b). There was no significant difference in overall survival between low and intermediate Revised International Prognostic Scoring System risk categories (data not shown).

Concerning treatment approach of all patients together, the overall survivals of patients treated with hypomethylating agents (median 18.3 months, $P=0.0002$ ) and stem cell transplant (median 31.5 months, $P<0.0001$ ) was significantly longer than patients receiving supportive care (median 6 months), but there was no significant difference in overall survivals of patients treated with induction chemotherapy (median 11.9 months, $P=0.20$ ) or lowintensity agents other than hypomethylating agents (median 8.8 months, $P=0.67$ ) compared to supportive care. In terms of treatment types given pre-stem cell transplant, there was no significant difference in overall survival of patients receiving supportive care or low-intensity agents other than hypomethylating agents (median 44.3 months), hypomethylating agents (median 32.5 months), or induction chemotherapy (median 26.1 months) $(P=0.43)$.

Among AEL patients only, the survival of patients with very-poor-risk karyotype (median 6.9 months) was significantly shorter than those with lower-risk karyotypes (median 22.8 months, $P<0.0001$ ) (Figure 2c). Patients with very high Revised International Prognostic Scoring System risk had a significantly shorter survival (median 10.5 months) than high (median 22.1 months, $P=0.02$ ) or low-intermediate (median 19.6 months, $P=0.03$ ) risk groups. There was no significant difference between survival of AEL patients with high (median 22.1 months) vs low-intermediate Revised International Prognostic Scoring System risk (median 19.6 months, $P=0.96$ ) (Figure 2d). There was no significant difference in the survival of AEL patients treated with induction chemotherapy compared to those treated with lower-intensity therapies or supportive care, whether including $(P=0.47$, Figure $3 a)$ or excluding $(P=0.67$, Figure $3 \mathrm{~b})$ patients who subsequently received stem cell transplant. Almost all AEL 

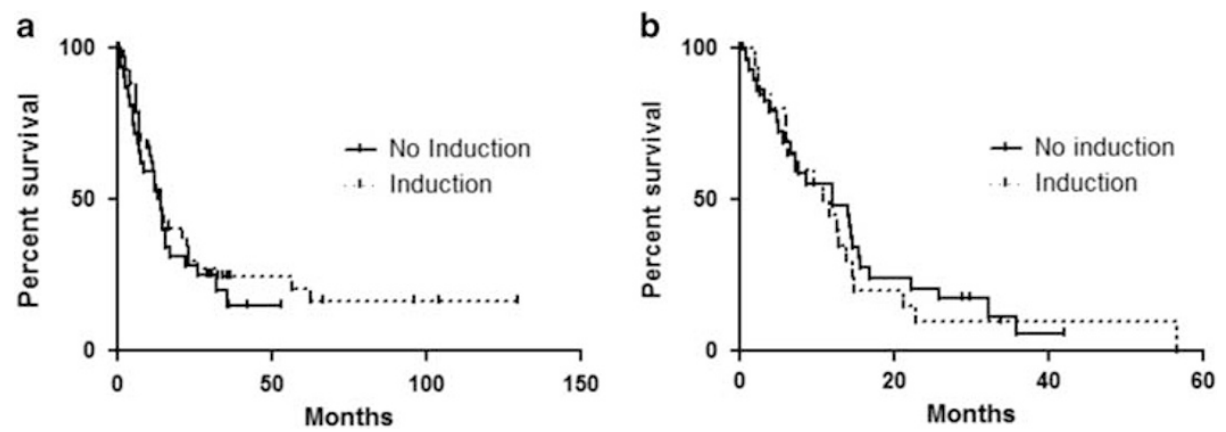

Figure 3 Overall survival of AEL patients according to the use of induction chemotherapy. (a) The overall survival of AEL patients treated with induction (median 13.9 months) is similar to those treated with lower intensity therapies or supportive care only (median 14.1 months, $P=0.47$ ). (b) Considering only the AEL patients who did not receive subsequent stem cell transplant ( $n=51$ ), the overall survival of those treated with induction (median 11.2 months) is similar to those treated with lower-intensity therapies or supportive care only (median 12.0 months, $P=0.67$ ).

patients receiving stem cell transplant had received prior induction chemotherapy (Table 2), thus we could not compare outcome in patients receiving different types of pre-stem cell transplant therapy. However, the three AEL patients who underwent stem cell transplant after treatment with hypomethylating agents only remain alive at 30.5, 36.2 , and 53.2 months after diagnosis. Given the differing treatment approaches between AEL and MDS, we compared overall survival of AEL with MDS patients within each treatment group. There were no significant differences of survival between AEL and MDS among patients treated with supportive care or low-intensity agents (excluding hypomethylating agents) only, hypomethylating agents, induction chemotherapy, or stem cell transplant, although there was a trend toward shorter survival in AEL patients receiving only supportive care/lowintensity agents $(P=0.08)$ (Figure 4$)$.

Multivariable Cox regression analysis was performed on the entire patient cohort, including factors significant to the level of $P<0.25$ in univariate analysis and the results are shown in Table 3. In the final model, very high and high Revised International Prognostic Scoring System risk groups were associated with shorter overall survival, while treatment with induction, hypomethylating agents, and stem cell transplant were each associated with longer overall survival. AEL diagnosis did not emerge as a significant variable in this analysis (Table 3).

\section{Discussion}

In this study, we performed a directed comparison of de novo acute erythroleukemia with primary refractory anemia with excess blasts that was further grouped by the presence or absence of erythroid predominance. We showed that although acute erythroleukemia was frequently associated with adverse cytogenetic abnormalities and TP53 mutation, these high-risk features were also common in refractory anemia with excess blasts. A diagnosis of acute erythroleukemia was associated with more frequent use of induction chemotherapy compared to refractory anemia with excess blasts, but induction chemotherapy did not appear to provide survival benefit for patients compared to lowerintensity therapies in retrospective analysis.

This study helps clarify a number of controversial issues that have surrounded acute erythroleukemia since the 2001 World Health Organization Classification redefined the disease as requiring $20 \%$ blasts among non-erythroid bone marrow cells. We showed that acute erythroleukemia bears many similarities to high-risk myelodysplastic syndrome. Clinically, most patients with acute erythroleukemia present with cytopenia(s) (often pancytopenia) rather than leukocytosis. As described in previous acute erythroleukemia studies, morphologic dysplasia is nearly ubiquitous in the erythroid lineage, ring sideroblasts are common, and dysplasia is also seen in megakaryocytes and granulocytes. ${ }^{8,16-18}$ The mutational profile of acute erythroleukemia, with common TP53 mutation (33\%) and uncommon FLT3 $(3 \%)$ and NPM1 (5\%) mutations, is different from most other acute myeloid leukemia subtypes, $, 9,10,19$ but similar to the distribution of these mutations in refractory anemia with excess blasts. The common cytogenetic abnormalities observed in acute erythroleukemia, such as -5/5q- and -7/7q-, are also frequent in de novo refractory anemia with excess blasts. However, we also observed some differences in acute erythroleukemia in comparison to patients with refractory anemia with excess blasts lacking erythroid predominance (MDS-typical): acute erythroleukemia patients presented with more severe cytopenias and had higher bone marrow and peripheral blood blasts and more frequent ring sideroblasts. Both acute erythroleukemia and refractory anemia with excess blasts and erythroid predominance (MDS-erythroid) demonstrated more frequent very-poor-risk cytogenetic abnormalities (including significantly more frequent $-7 /$ del7q in MDS-erythroid) compared to MDS-typical, which further confirms recent findings that erythroid-rich 


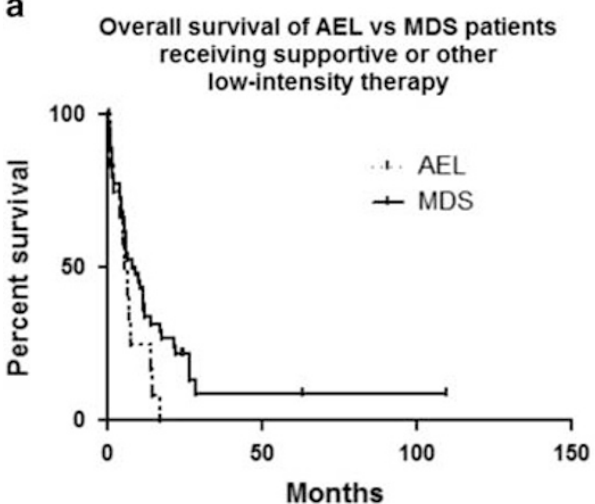

C

Overall survival of AEL vs MDS patients treated with induction chemotherapy

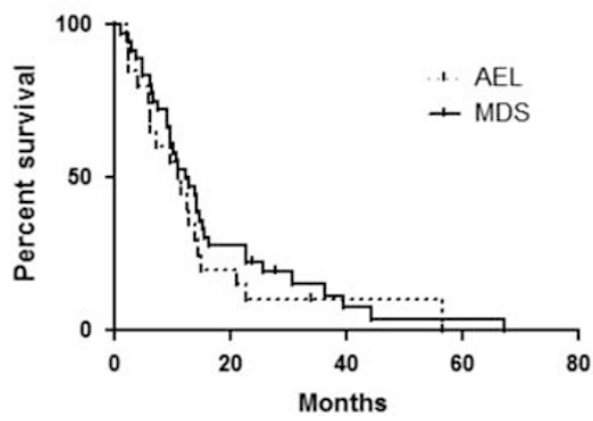

b

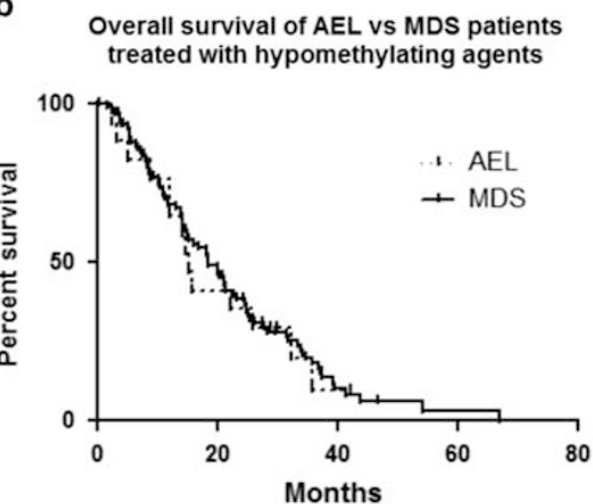

d

Overall survival of AEL vs MDS patients treated with stem cell transplant

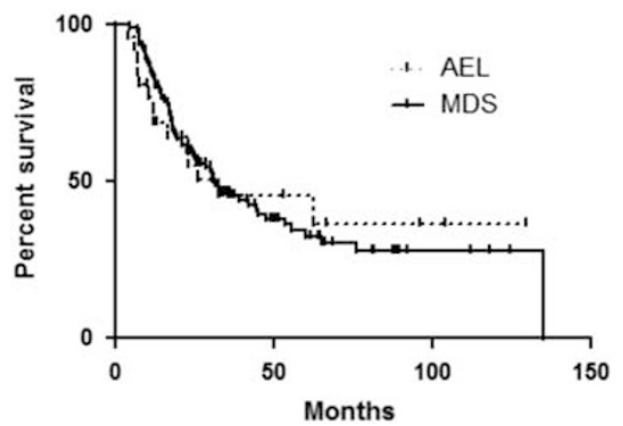

Figure 4 Comparison of overall survival of acute erythroleukemia (AEL) versus refractory anemia with excess blasts patients (MDS) according to therapy administered. (a) AEL patients treated with only supportive care or low-intensity therapies other than hypomethylating agents (without subsequent stem cell transplant) have a borderline shorter overall survival (median 6.2 months) compared to similarly treated MDS patients (median 9.0 months, $P=0.08$ ). (b) AEL patients treated with hypomethylating agents (without subsequent induction therapy or stem cell transplant) have a similar overall survival (median 15.3 months) to similarly treated MDS patients (median 18.5 months, $P=0.84$ ). (c) AEL patients treated with induction chemotherapy (without subsequent stem cell transplant) have a similar overall survival (median 11.2 months) to similarly treated MDS patients (median 12.6 months, $P=0.40$ ). (d) AEL patients treated with stem cell transplant have a similar overall survival (median 32.4 months) to similarly treated MDS patients (median 31.5 months, $P=0.99$ ).

Table 3 Multivariable analysis of factors influencing overall survival in combined AEL and MDS group patients ( $n=356$ )

\begin{tabular}{lcr}
\hline Risk factor at diagnosis & P value & HR [95\% CI] \\
\hline Age (per year increase) & 0.086 & $1.010[0.999-1.022]$ \\
Acute erythroleukemia diagnosis & 0.298 & $1.168[0.882-1.566]$ \\
IPSS-R very-high-risk group $^{\mathrm{a}}$ & $<0.0001$ & $3.147[2.280-4.345]$ \\
IPSS-R high-risk group $^{\mathrm{a}}$ & $<0.005$ & $1.633[1.162-2.294]$ \\
Treatment with hypomethylating agents, without stem cell transplant $^{\mathrm{b}}$ & 0.0001 & $0.420[0.294-0.601]$ \\
Treatment with induction chemotherapy, without stem cell transplant $^{\mathrm{b}}$ & $<0.0001$ & $0.559[0.367-0.850]$ \\
Treatment with stem cell transplant $^{\mathrm{b}}$ & $0.207[0.136-0.315]$
\end{tabular}

Abbreviation: IPSS-R, Revised International Prognostic Scoring System.

${ }^{\mathrm{a} C o m p a r e d ~ t o ~ I P S S-R ~ L o w / i n t e r m e d i a t e ~ c o m b i n e d ~ g r o u p . ~}$

${ }^{\mathrm{b}}$ Compared to supportive care or low-intensity treatments other than hypomethylating agents.

refractory anemia with excess blasts and acute erythroleukemia have a similar prognosis regardless of blast count and that karyotype risk assessment provides better prognostic stratification than blast count across the spectrum of erythroid-rich myeloid neoplasms. ${ }^{4,8,17}$

The underlying biology of the marked erythroid proliferations in erythroid-rich refractory anemia with excess blasts and acute erythroleukemia is not well understood. As shown previously, it is not likely due to exogenous erythropoiesis stimulating agent administration ${ }^{20}$ or endogenous erythropoietin production. ${ }^{4}$ Specific mutations that may underlie erythroid proliferation in this setting have not been identified, and we did not find differences in the mutational profile between AEL, MDS-erythroid, 
and MDS-typical groups in the genes we tested. Acute erythroleukemia cases often had a very complex karyotype with chromosome 5 and 7 abnormalities and these cases were frequently associated with TP53 mutations. The combination of a very complex karyotype, TP53 mutation, and increased erythroid proliferation also characterizes pure erythroid leukemia ${ }^{18,21}$ and is frequently seen in therapy-related myelodysplastic syndrome, ${ }^{8,14,22}$ both of which are high-grade myeloid neoplasms with an extremely poor prognosis. These findings raise the possibility that in high-risk acute erythroleukemia, complex genetic alterations may drive erythroid proliferation and severely impair myeloid maturation and differentiation, leading to clinical aggressiveness.

On univariate analysis, there was a trend toward shorter overall survival of AEL patients compared to MDS-typical patients, but this did not reach statistical significance $(P=0.14)$. In multivariable analysis, acute erythroleukemia was not an independent risk factor when the Revised International Prognostic Scoring System score was included. AEL patients had a higher frequency of very-poor-risk karyotypes compared to MDS-typical patients and AEL patients with very-poor-risk karyotype had short survival (median overall survival of 6.9 months). Wells et $a 1^{23}$ also reported significantly shorter survival for acute erythroleukemia patients with higher-risk karyotypes compared to standard-risk cytogenetics. The 2012 Revised International Prognostic Scoring System scheme, unlike the original 1997 International Prognostic Scoring System, divides complex karyotypes into those with three (poor risk) or more than three (very poor risk) independent abnormalities. ${ }^{12}$ The adverse cytogenetic characteristics of acute erythroleukemia were appropriately captured by the Revised International Prognostic Scoring System cytogenetic risk categorization. Interestingly, we found no significant difference in outcome in either the AEL group or in the entire cohort between karyotype risk groups other than very poor risk, underscoring the importance of the new very-poor-risk karyotype category in stratifying both acute erythroleukemia and refractory anemia with excess blasts. Using the Revised International Prognostic Scoring System, ${ }^{24}$ more AEL group patients were assigned a very high total score compared to MDS-typical group patients. The very-high-risk AEL patients had a significantly shorter survival than other groups; we did not find a significant difference in survival between the low-intermediate and high-risk groups, which may have been due to a relatively small number of AEL patients in the low-intermediate risk groups.

We also showed that a much higher proportion of patients diagnosed with acute erythroleukemia received induction chemotherapy compared to refractory anemia with excess blasts. The European LeukemiaNet guidelines on primary myelodysplastic syndrome recommend reserving induction chemotherapy for fit myelodysplastic syndrome patients without a suitable donor who are younger than $65-70$ years and have $10 \%$ or more bone marrow blasts, without adverse cytogenetic characteristics; only $10 / 77$ of the acute erythroleukemia patients in our series (13\%) would have met criteria for this guideline. The overall survival of acute erythroleukemia patients in our series was similar whether or not the patients received induction therapy, and when stratified by treatment modalities was similar to refractory anemia with excess blasts, with the exception of a borderline shorter overall survival in acute erythroleukemia patients treated with only supportive care or low-intensity therapies. These results, although derived from a retrospective study, are similar to the randomized phase III trial in high-risk myelodysplastic syndrome with up to $30 \%$ blasts $^{25,26}$ that reported a survival benefit with azacitidine over conventional treatment. Grossmann et al also reported a poor outcome with acute erythroleukemia patients treated with standard induction chemotherapy regimens, with a median overall survival of 9.3 months for non-NPM1-mutated cases, but a much better outcome for patients with intermediate karyotype risk, particularly those with NPM1 mutation (16\% of cases). ${ }^{10}$ Although NPM1 mutation is currently not acute myeloid leukemiadefining, myeloid neoplasms with $<20 \%$ blasts and NPM1 mutation appear to rapidly progress to acute myeloid leukemia ${ }^{27}$ and such patients may benefit from induction chemotherapy. Given the few patients with NPM1 mutation in our patient cohort, we could not address this question in our study.

For pre-stem cell transplant cytoreduction, the majority of acute erythroleukemia patients (88\%) received induction chemotherapy, compared to only $9 \%$ of refractory anemia with excess blasts patients who had not progressed to acute myeloid leukemia. Despite different pre-stem cell transplant cytoreduction regimens, acute erythroleukemia and refractory anemia with excess blasts patients treated with stem cell transplant had similar outcomes. Only three of our acute erythroleukemia patients received stem cell transplant without induction chemotherapy (all treated with hypomethylating agents), and all three patients are alive 30.5-53.2 months after diagnosis. Similar findings have been reported in myelodysplastic syndrome in that patients receiving prestem cell transplant hypomethylating agents vs induction chemotherapy showed a similar disease relapse rates, non-relapse mortality, event-free survival, and overall survival. ${ }^{28,29}$

In summary, our data show that compared to refractory anemia with excess blasts, patients with de novo acute erythroleukemia have a higher frequency of adverse cytogenetic abnormalities, more severe cytopenias at presentation, and are more frequently treated with intensive induction chemotherapy, but exhibit a similar clinical outcome across various therapeutic approaches. These data support the updated 4th edition World Health 
Organization Classification of Myeloid Neoplasms reclassification of acute erythroleukemia, erythroid/ myeloid subtype with $<20 \%$ blasts as refractory anemia with excess blasts rather than as a subtype of acute myeloid leukemia. ${ }^{30}$ The biological factors that underlie the erythroid predominance and ineffective erythropoiesis in acute erythroleukemia and erythroid-rich refractory anemia with excess blasts compared to other acute myeloid leukemia and refractory anemia with excess blasts without erythroid predominance are unknown, but prognostic differences within acute erythroleukemia appear to be adequately captured by the Revised International Prognostic Scoring System scheme. Of note, as with all myeloid neoplasms with $<20 \%$ blasts, careful exclusion of genetically categorized entities such as acute myeloid leukemia with $t(15 ; 17), \operatorname{inv}(16) / t(16 ; 16)$, or $t(8 ; 21), t(9 ; 11)$, or $t(1 ; 22)$ is required; however, such cases are exceedingly rare in erythroid-rich myeloid neoplasms, and none were present in our search of acute myeloid leukemia and myelodysplastic syndrome cases with $>50 \%$ erythroids at three large institutions (data not shown). Our data, although retrospective, support a riskstratification and treatment approach to acute erythroleukemia that is similar to refractory anemia with excess blasts that is based on the standard risk factors used to evaluate myelodysplastic syndrome patients: blast percentage of all marrow cells, peripheral blood counts, and karyotype risk. The role of molecular genetic studies, particularly NPM1 mutation, in acute erythroleukemia risk stratification (as well as myelodysplastic syndromes in general) requires further study.

\section{Acknowledgments}

Sa Wang and Robert Hasserjian designed the research study, performed the research, analyzed the data, and wrote the paper. Keyur Patel, Jie Peng, Zhuang Zho, and Paola Dal Cin contributed cytogenetic and molecular genetic analysis, and Olga Pozdnyakova and David Steensma analyzed the data and contributed to writing of the paper. All authors have reviewed the final submitted version of this manuscript.

\section{Disclosure/conflict of interest}

The authors declare no conflict of interest.

\section{References}

1 Harris NL, Jaffe ES, Diebold J et al. World Health Organization classification of neoplastic diseases of the hematopoietic and lymphoid tissues: report of the Clinical Advisory Committee Meeting-Airlie House, Virginia, November 1997. J Clin Oncol 1999;17: 3835-3849.
2 Bennett JM, Catovsky D, Daniel MT et al. Proposed revised criteria for the classification of acute myeloid leukemia: a report of the French-AmericanBritish Cooperative Group. Ann Intern Med 1985;103: 620-625.

3 Senent L, Arenillas L, Luno E et al. Reproducibility of the World Health Organization 2008 criteria for myelodysplastic syndromes. Haematologica 2013;98: 568-575.

4 Wang SA, Tang G, Fadare O et al. Erythroid-predominant myelodysplastic syndromes: enumeration of blasts from nonerythroid rather than total marrow cells provides superior risk stratification. Mod Pathol 2008;21:1394-1402.

5 Santos FP, Faderl S, Garcia-Manero G et al. Adult acute erythroleukemia: an analysis of 91 patients treated at a single institution. Leukemia 2009;23:2275-2280.

6 Peng J, Hasserjian RP, Tang G et al. Myelodysplastic syndromes following therapy with hypomethylating agents (HMAs): development of acute erythroleukemia may not influence assessment of treatment response. Leuk Lymphoma 2016;57:812-829.

7 Wang SA, Hasserjian RP. Acute erythroleukemias, acute megakaryoblastic leukemias, and reactive mimics: a guide to a number of perplexing entities. Am J Clin Pathol 2015;144:44-60.

8 Kasyan A, Medeiros LJ, Zuo Z et al. Acute erythroid leukemia as defined in the World Health Organization classification is a rare and pathogenetically heterogeneous disease. Mod Pathol 2010;23:1113-1126.

9 Zuo Z, Medeiros LJ, Chen Z et al. Acute myeloid leukemia (AML) with erythroid predominance exhibits clinical and molecular characteristics that differ from other types of AML. PloS ONE 2012;7:e41485.

10 Grossmann V, Bacher U, Haferlach C et al. Acute erythroid leukemia (AEL) can be separated into distinct prognostic subsets based on cytogenetic and molecular genetic characteristics. Leukemia 2013;27: 1940-1943.

11 Burnett AK, Milligan D, Prentice AG et al. A comparison of low-dose cytarabine and hydroxyurea with or without all-trans retinoic acid for acute myeloid leukemia and high-risk myelodysplastic syndrome in patients not considered fit for intensive treatment. Cancer 2007;109:1114-1124.

12 Schanz J, Tuchler H, Sole F et al. New comprehensive cytogenetic scoring system for primary myelodysplastic syndromes (MDS) and oligoblastic acute myeloid leukemia after MDS derived from an international database merge. J Clin Oncol 2012;30:820-829.

13 Ok CY, Patel KP, Garcia-Manero G et al. Mutational profiling of therapy-related myelodysplastic syndromes and acute myeloid leukemia by next generation sequencing, a comparison with de novo diseases. Leuk Res 2015;39:348-354.

14 Ok CY, Patel KP, Garcia-Manero G et al. TP53 mutation characteristics in therapy-related myelodysplastic syndromes and acute myeloid leukemia is similar to de novo diseases. J Hematol Oncol 2015;8:45.

15 Cleven AH, Nardi V, Ok CY et al. High p53 protein expression in therapy-related myeloid neoplasms is associated with adverse karyotype and poor outcome. Mod Pathol 2015;28:552-563.

16 Olopade OI, Thangavelu M, Larson RA et al. Clinical, morphologic, and cytogenetic characteristics of 26 patients with acute erythroblastic leukemia. Blood 1992;80:2873-2882. 
17 Hasserjian RP, Zuo Z, Garcia C et al. Acute erythroid leukemia: a reassessment using criteria refined in the 2008 WHO classification. Blood 2010;115:1985-1992.

18 Liu W, Hasserjian RP, Hu Y et al. Pure erythroid leukemia: a reassessment of the entity using the 2008 World Health Organization classification. Mod Pathol 2011;24:375-383.

19 Bacher U, Haferlach C, Alpermann T et al. Comparison of genetic and clinical aspects in patients with acute myeloid leukemia and myelodysplastic syndromes all with more than $50 \%$ of bone marrow erythropoietic cells. Haematologica 2011;96:1284-1292.

20 Hellstrom-Lindberg E, Kanter-Lewensohn L, Ost A. Morphological changes and apoptosis in bone marrow from patients with myelodysplastic syndromes treated with granulocyte-CSF and erythropoietin. Leuk Res 1997;21:415-425.

21 Wong E, Ling V, Westerman D, Morgan S, Juneja S. How unique is pure erythroid leukaemia? A retrospective analysis of seven cases and review of the literature. J Clin Pathol 2015;68:301-305.

22 Czader M, Orazi A. Therapy-related myeloid neoplasms. Am J Clin Pathol 2009;132:410-425.

23 Wells AW, Bown N, Reid MM et al. Erythroleukaemia in the north of England: a population based study. J Clin Pathol 2001;54:608-612.

24 Greenberg PL, Tuechler H, Schanz J et al. Revised international prognostic scoring system for myelodysplastic syndromes. Blood 2012;120:2454-2465.
25 Fenaux P, Mufti GJ, Hellstrom-Lindberg E et al. Efficacy of azacitidine compared with that of conventional care regimens in the treatment of higherrisk myelodysplastic syndromes: a randomised, open-label, phase III study. Lancet Oncol 2009;10: 223-232.

26 Fenaux P, Mufti GJ, Hellstrom-Lindberg E et al. Azacitidine prolongs overall survival compared with conventional care regimens in elderly patients with low bone marrow blast count acute myeloid leukemia. J Clin Oncol 2010;28:562-569.

27 Peng J, Zuo Z, Fu B et al. Chronic myelomonocytic leukemia with nucleophosmin (NPM1) mutation. Eur J Haematol 2016;96:65-71.

28 Damaj G, Duhamel A, Robin $M$ et al. Impact of azacitidine before allogeneic stem-cell transplantation for myelodysplastic syndromes: a study by the Societe Francaise de Greffe de Moelle et de Therapie-Cellulaire and the Groupe-Francophone des Myelodysplasies. J Clin Oncol 2012;30:4533-4540.

29 Gerds AT, Gooley TA, Estey EH et al. Pretransplantation therapy with azacitidine vs induction chemotherapy and posttransplantation outcome in patients with MDS. Biol Blood Marrow Transplant 2012;18: 1211-1218.

30 Arber DA, Orazi A, Hasserjian R et al. The 2016 revision to the World Health Organization (WHO) classification of myeloid neoplasms and acute leukemia. Blood 2016;127:2391-2405. 